\title{
WARPED PRODUCT SEMI-SLANT SUBMANIFOLDS IN LOCALLY RIEMANNIAN PRODUCT MANIFOLDS
}

\author{
MEHMET ATÇEKEN
}

(Received 27 March 2007)

\begin{abstract}
In this paper, we prove that there are no warped product proper semi-slant submanifolds such that the spheric submanifold of a warped product is a proper slant. But we show by means of examples the existence of warped product semi-slant submanifolds such that the totally geodesic submanifold of a warped product is a proper slant submanifold in locally Riemannian product manifolds.
\end{abstract}

2000 Mathematics subject classification: 53C15, 53C40.

Keywords and phrases: warped product, slant distribution, semi-slant submanifold, spheric foliation and locally Riemannian product manifold.

\section{Introduction}

The differential geometry of slant submanifolds has shown an increasing development since B.-Y. Chen defined slant immersion in complex geometry as a natural generalization of both holomorphic and totally real immersions [2-6].

In [7], Lotto introduced the notion of slant immersion of a Riemannian manifold into an almost contact metric manifold. Recently, in [12], Li and Li defined and studied the geometry of a semi-slant submanifold in locally Riemannian product manifolds. The class of proper semi-slant submanifolds appears as a particular case of the class of warped product semi-slant submanifolds because the class of proper semiinvariant submanifolds is a particular case of the proper warped product semi-invariant submanifolds.

Let $M$ be an $m$-dimensional manifold with a tensor of type $(1,1)$ such that $F^{2}=I$ and $F \neq \pm I$. Then $M$ is said to be an almost product manifold with almost product structure $F$. If an almost product manifold $M$ has a Riemannian metric $g$ such that $g(F X, Y)=g(X, F Y)$, for any $X, Y \in \Gamma(T M)$, then $M$ is called an almost Riemannian product manifold. We denote the Levi-Civita connection on $M$ by $\bar{\nabla}$ with respect to $g$. If $\left(\bar{\nabla}_{X} F\right) Y=0$, for any $X, Y \in \Gamma(T M)$, then $M$ is called a locally Riemannian product manifold [12].

(C) 2008 Australian Mathematical Society 0004-9727/08 \$A2.00 + 0.00 
Let $M$ be a Riemannian manifold with almost Riemannian product structure $F$ and let $N$ be an isometrically immersed submanifold in $M$. For each $x \in N$, we denote by $D_{x}$ the maximal invariant subspace of the tangent space $T_{x} N$ of $N$. If the dimension of $D_{x}$ is the same for all $x$ in $N$, then $D_{x}$ gives an invariant distribution $D$ on $N$.

A submanifold $N$ in a locally Riemannian product manifold is called a semiinvariant submanifold if there exists on $N$ a differentiable invariant distribution $D$ whose orthogonal complement $D^{\perp}$ is an anti-invariant distribution, that is, $F\left(D^{\perp}\right) \subset T N^{\perp}$. A semi-invariant submanifold is called an anti-invariant (invariant) submanifold if $\operatorname{dim}\left(D_{x}\right)=0\left(\operatorname{dim}\left(D_{x}^{\perp}\right)=0\right)$. On the other hand, it is called proper semi-invariant if it is neither invariant nor anti-invariant.

A semi-invariant submanifold in the form $N=N_{T} \times N_{\perp}$ of a locally Riemannian product manifold $M$ is called a Riemannian product if $N_{T}$ and $N_{\perp}$ are totally geodesic submanifolds of $N$, where $N_{T}$ is an invariant submanifold and $N_{\perp}$ is an anti-invariant submanifold of $M$. The notion of semi-invariance in a locally Riemannian product manifold was introduced in $[1,9,11]$.

The above definitions have been generalized as follows.

(1) The submanifold $N$ is called a semi-invariant submanifold if there exists a differentiable distribution $D: x \longrightarrow D_{x} \subset T_{x} N$ such that $D$ is invariant and the complementary distribution $D^{\perp}$ is anti-invariant distribution.

(2) The submanifold $N$ is called a slant submanifold if for each nonzero vector field $X \in \Gamma(T N)$, the angle $\theta(x)$ between $F X$ and $T_{x} N$ is constant, that is, it does not dependent on of the choice $x \in N$ and $X \in \Gamma\left(T_{x} N\right)$.

(3) The submanifold $N$ is referred to as semi-slant if it has two orthogonal distributions such as $D$ and $D^{\prime}$ such that $D$ is an invariant distribution and $D^{\prime}$ is a slant distribution.

It is well known that the notion of warped products plays an important role in differential geometry as well as in physics. For a recent survey on warped products as Riemannian submanifolds, we refer to [4, 5, 8].

Let $N_{1}$ and $N_{2}$ be two Riemannian manifolds with Riemannian metrics $g_{1}$ and $g_{2}$, respectively, and $f$ be differentiable function on $N_{1}$. Consider the product manifold $N_{1} \times N_{2}$ with its projection $\pi: N_{1} \times N_{2} \longrightarrow N_{1}$ and $\eta: N_{1} \times N_{2} \longrightarrow N_{2}$. The warped product manifold $N=N_{1} \times{ }_{f^{2}} N_{2}$ is the manifold $N_{1} \times N_{2}$ equipped with the Riemannian metric structure such that

$$
\|X\|^{2}=\left\|\pi_{*} X\right\|^{2}+f^{2}(\pi(x))\left\|\eta_{*} X\right\|^{2},
$$

for any $X \in \Gamma(T N)$. Thus we have $g=g_{1}+f^{2} g_{2}$, where $f$ is called the warping function of the warped product. The warped product manifold $N=N_{1} \times f_{f^{2}} N_{2}$ is characterized by the fact that $N_{1}$ and $N_{2}$ are totally geodesic and spheric foliations of $N$, respectively. If the warping function is constant, a warped product is said to be the Riemannian product [10].

The purpose of this paper is to investigate a new class of submanifolds of locally Riemannian product manifolds, that is, warped product semi-slant submanifolds. 
We shall focus our attention mainly on warped product semi-slant submanifolds which contain warped product semi-invariant submanifolds and Riemannian product semislant submanifolds as a general case.

\section{Preliminaries}

If $N$ is an isometrically immersed submanifold in a Riemannian manifold $M$, then the formulas of Gauss and Weingarten for $N$ in $M$ are given, respectively, by

$$
\bar{\nabla}_{X} Y=\nabla_{X} Y+h(X, Y)
$$

and

$$
\bar{\nabla}_{X} V=-A_{V} X+\nabla_{X}^{\perp} V
$$

for any $X, Y \in \Gamma(T N)$ and $V \in \Gamma\left(T N^{\perp}\right)$, where $\bar{\nabla}$ and $\nabla$ denote the Riemannian connections on $M$ and $N$, respectively, $h$ is the second fundamental form of $N$ in $M$, $\nabla^{\perp}$ is the normal connection on the normal bundle and $A$ is the shape operator of $N$ in $M$. The second fundamental form and the shape operator are related by

$$
g\left(A_{V} X, Y\right)=g((h(X, Y), V),
$$

where $g$ denotes the Riemannian metric on $M$ as well as $N$. For any a submanifold $N$ of a Riemannian manifold $M$, Gauss's equation is given by

$$
\bar{R}(X, Y) Z=R(X, Y) Z+A_{h(X, Z)} Y-A_{h(Y, Z)} X+\left(\bar{\nabla}_{X} h\right)(Y, Z)-\left(\bar{\nabla}_{Y} h\right)(X, Z),
$$

for any $X, Y, Z \in \Gamma(T N)$, where $\bar{R}$ and $R$ denote the Riemannian curvature tensors of $M$ and $N$, respectively. The covariant derivative of $h$ is defined by

$$
\left(\bar{\nabla}_{X} h\right)(Y, Z)=\nabla_{X}^{\perp} h(Y, Z)-h\left(\nabla_{X} Y, Z\right)-h\left(\nabla_{X} Z, Y\right) .
$$

We recall the following general lemma from [10] for later use.

LEMMA 2.1. Let $N=N_{1} \times_{f} N_{2}$ be a warped product manifold with warping function $f$. Then:

(1) $\nabla_{X} Y \in \Gamma\left(T N_{1}\right)$ for each $X, Y \in \Gamma\left(T N_{1}\right)$;

(2) $\nabla_{X} Z=\nabla_{Z} X=X(\ln f) Z$, for each $X \in \Gamma\left(T N_{1}\right), Z \in \Gamma\left(T N_{2}\right)$;

(3) $\nabla_{Z} W=\nabla_{Z}^{N_{2}} W-g(Z, W)(($ gradf $) / f)$, for each $Z, W \in \Gamma\left(T N_{2}\right)$.

Here $\nabla$ and $\nabla^{N_{2}}$ denote the Levi-Civita connections on $N$ and $N_{2}$, respectively.

\section{Warped product semi-slant submanifolds of a locally Riemannian product manifold}

Now, let $N=N_{1} \times_{f} N_{2}$ be an immersed submanifold of a locally Riemannian product manifold $M$ and denote the orthogonal complementary of $F(T N)$ in $T N^{\perp}$ by $V$. Then we have the direct sum

$$
T N^{\perp}=F(T N) \oplus V .
$$


We can easily see that $V$ is an invariant sub-bundle with respect to $F$. Furthermore, for any nonzero vector $X$ tangent to $N$, we put

$$
F X=T X+\omega X
$$

where $T X$ and $\omega X$ denote the tangential and normal components of $F X$, respectively. For each nonzero vector $X$ tangent to $N$ at $x$, the angle $\theta(x), 0 \leq \theta(x) \leq(\pi / 2)$, between $F X$ and $T_{x} N$ is called the slant angle. If the slant angle is constant, then the submanifold is also called the slant submanifold. Invariant and antiinvariant submanifolds are slant submanifolds with slant angle $\theta=0$ and $\theta=(\pi / 2)$, respectively. A slant submanifold is said to be proper slant if it is neither invariant nor anti-invariant.

In the same way, for any vector $V$ normal to $N$, we put

$$
F V=t V+n V
$$

where $t V$ and $n V$ denote the tangential and normal components of $F V$, respectively.

THEOREM 3.1. Let $N$ be a submanifold of a locally Riemannian product manifold $M$. Then $N$ is a slant submanifold if and only if there exists a constant $\lambda \in[0,1]$ such that $T^{2}=\lambda I$. In this case, if $\theta$ is the slant angle of $N$, then it satisfies $\lambda=\cos ^{2} \theta$ [12].

DEFinition 3.1. $N$ is called a semi-slant submanifold of a locally Riemannian product manifold $M$ if there exist two orthogonal distributions such as $D$ and $D^{\prime}$ such that:

(1) $T N$ has the orthogonal direct $\operatorname{sum} T N=D \oplus D^{\prime}$;

(2) the distribution $D$ is an invariant distribution, that is, $F(D)=D$;

(3) the distribution $D^{\prime}$ is a slant with angle $\theta \neq 0$ and $\theta \neq(\pi / 2)$ [2].

THEOREM 3.2. Let $D$ be a distribution on $N$. Then $D$ is a slant distribution if and only if there exists a constant $\lambda \in[0,1]$ such that $\left(P_{1} T\right)^{2} X=\lambda X$ for any $X \in \Gamma(D)$. In this case, if $\theta$ is the slant angle of $D$, then it satisfies $\lambda=\cos ^{2} \theta$, where $P_{1}$ denotes the orthogonal projection on $D$ [12].

Furthermore, if $N$ is a slant submanifold of a locally Riemannian product manifold $M$ with slant angle $\theta$, then

$$
g(T X, T Y)=\cos ^{2} \theta g(X, Y) \quad \text { and } \quad g(\omega X, \omega Y)=\sin ^{2} \theta g(X, Y),
$$

for any $X, Y \in \Gamma(T N)$.

In this section, we study warped product semi-slant submanifolds, with warped product in the form $N=N_{1} \times_{f} N_{2}$, in a locally Riemannian product manifold $M$. First, we suppose that $N_{1}$ is an invariant and $N_{2}$ is a semi-slant of $M$ with slant angle $\theta \neq(\pi / 2), 0$. Later, $N_{1}$ will be an anti-invariant submanifold and $N_{2}$ will be a semislant submanifold of $M$ with respect to $F$. 
THEOREM 3.3. Let $M$ be a locally Riemannian product manifold and $N$ be a submanifold of $M$. Then there exist no warped product semi-slant submanifolds $N=N_{T} \times{ }_{f} N_{\theta}$ in $M$ such that $N_{T}$ is an invariant submanifold and $N_{\theta}$ is a proper slant submanifold of $M$.

Proof. We suppose that $N=N_{T} \times{ }_{f} N_{\theta}$ is a warped product proper semi-slant submanifold of a locally Riemannian product manifold $M$ such that $N_{T}$ is invariant and $N_{\theta}$ is a proper slant submanifold of $M$. We denote the projections onto $\Gamma\left(T N_{T}\right)$ and $\Gamma\left(T N_{\theta}\right)$ by $P_{1}$ and $P_{2}$, respectively. Then for any vector $Z \in \Gamma(T N)$, we can put

$$
Z=P_{1} Z+P_{2} Z
$$

and using (7) gives

$$
F Z=F P_{1} Z+F P_{2} Z=T P_{1} Z+T P_{2} Z+\omega P_{2} Z .
$$

By using the Gauss-Weingarten formulas, (7), (8) and considering Lemma 2.1(2) we obtain

$$
\begin{aligned}
\bar{\nabla}_{U} F X= & F \bar{\nabla}_{U} X, \\
T X \ln (f) U+h(U, T X)= & X \ln (f) T P_{2} U+X \ln (f) \omega P_{2} U \\
& +\operatorname{th}(U, X)+n h(U, X),
\end{aligned}
$$

for any $X \in \Gamma\left(T N_{T}\right)$ and $U \in \Gamma\left(T N_{\theta}\right)$. Then, comparing tangential and normal components in (12) respectively, we obtain

$$
T X \ln (f) U=X \ln (f) T P_{2} U+\operatorname{th}(U, X)
$$

and

$$
h(U, T X)=X \ln (f) \omega P_{2} U+n h(U, X) .
$$

In the same way, we arrive at

$$
\begin{aligned}
\bar{\nabla}_{X} F U & =F \bar{\nabla}_{X} U, \\
\bar{\nabla}_{X} T P_{2} U+\bar{\nabla}_{X} \omega P_{2} U & =F \nabla_{X} U+F h(U, X), \\
\nabla_{X} T P_{2} U+h\left(X, T P_{2} U\right)-A_{\omega P_{2} U} X+\nabla_{X}^{\perp} \omega P_{2} U & =F(X \ln (f) U)+F h(X, U) \\
= & X \ln (f) T P_{2} U \\
& +X \ln (f) \omega P_{2} U \\
& +\operatorname{th}(X, U)+n h(X, U),
\end{aligned}
$$

for any $X \in \Gamma\left(T N_{T}\right)$ and $U \in \Gamma\left(T N_{\theta}\right)$. Taking into account the tangential and normal components of (15) respectively, we obtain

$$
A_{\omega P_{2} U} X=-\operatorname{th}(U, X)
$$


and

$$
h\left(X, T P_{2} U\right)+\nabla_{X}^{\perp} \omega P_{2} U=X \ln (f) \omega P_{2} U+n h(X, U) .
$$

By using (3) and (16), it is easily seen that

$$
\begin{aligned}
g\left(A_{\omega P_{2} U} X, U\right)=- & g(t h(U, X), U)=-g(F h(U, X), U)=-g(h(U, X), F U), \\
& g\left(h(U, X), \omega P_{2} U\right)=-g\left(h(U, X), \omega P_{2} U\right),
\end{aligned}
$$

that is,

$$
g\left(h(U, X), \omega P_{2} U\right)=0 .
$$

On the other hand, replacing $X$ by $T X$ in (14) and taking into account $T N_{T}$ being invariant, we obtain

$$
\begin{aligned}
T X \ln (f) g\left(\omega P_{2} U, \omega P_{2} U\right) & =g\left(h(U, X)-n h(U, T X), \omega P_{2} U\right) \\
& =g\left(h(U, X), \omega P_{2} U\right)-g\left(n h(U, X), \omega P_{2} U\right) \\
& =g\left(h(U, X), \omega P_{2} U\right)=0,
\end{aligned}
$$

for any $X \in \Gamma\left(T N_{T}\right)$ and $U \in \Gamma\left(T N_{\theta}\right)$. Thus,

$$
T X \ln (f) \sin ^{2} \theta g\left(P_{2} U, P_{2} U\right)=0 .
$$

Since $N_{\theta}$ is a proper slant submanifold, $g$ is a Riemannian metric and $P_{2} U$ is a nonnull vector, we arrive at $T X \ln (f)=0$, that is, the warping function $f$ is constant. Hence, the proof is complete.

THEOREM 3.4. Let $M$ be a locally Riemannian product manifold and $N$ be a submanifold of $M$. Then there exist no warped product semi-slant submanifolds $N=N_{\perp} \times{ }_{f} N_{\theta}$ in $M$ such that $N_{\perp}$ is an anti-invariant submanifold and $N_{\theta}$ is a proper slant submanifold of $M$.

PROOF. We suppose that $N=N_{\perp} \times_{f} N_{\theta}$ is a warped product semi-slant submanifold such that $N_{\perp}$ is an anti-invariant submanifold and $N_{\theta}$ is a proper slant submanifold of a locally Riemannian product manifold $M$. Then for any vectors $X, Y$ tangent to $N_{\perp}$ and $U$ tangent to $N_{\theta}$,

$$
\begin{gathered}
\bar{\nabla}_{U} F X=F \bar{\nabla}_{U} X \\
-A_{\omega X} U+\nabla_{U}^{\perp} \omega X=F(X \ln (f) U)+\operatorname{th}(U, X)+n h(U, X) .
\end{gathered}
$$

From the tangential components of (19), we obtain

$$
-A_{\omega X} U=X \ln (f) T P_{2} U+\operatorname{th}(U, X) .
$$


Furthermore, from equations (1), (2), (7), (8), (11) and considering Lemma 2.1,

$$
\begin{aligned}
\bar{\nabla}_{X} F U & =F \bar{\nabla}_{X} U, \\
\bar{\nabla}_{X} T P_{2} U+\bar{\nabla}_{X} \omega P_{2} U= & F \nabla_{X} U+F h(X, U), \\
\nabla_{X} T P_{2} U+h\left(X, T P_{2} U\right)-A_{\omega P_{2} U} X+\nabla_{X}^{\perp} \omega P_{2} U= & F(X \ln (f) U)+\operatorname{th}(X, U) \\
& +n h(X, U) \\
= & X \ln (f) T P_{2} U \\
& +X \ln (f) \omega P_{2} U \\
& +\operatorname{th}(X, U)+n h(X, U) .
\end{aligned}
$$

From the tangential components of (21),

$$
A_{\omega P_{2} U} X=-\operatorname{th}(X, U)
$$

In the same way, making use of (1), (2), taking account of $N_{\perp}$ being anti-invariant in $M$ and totally geodesic in $N$, we obtain

$$
\begin{gathered}
\bar{\nabla}_{Y} F X=F \bar{\nabla}_{Y} X, \\
-A_{\omega X} Y+\nabla_{Y}^{\perp} \omega X=F \nabla_{Y} X+\operatorname{th}(X, Y)+n h(X, Y),
\end{gathered}
$$

which gives

$$
A_{\omega X} Y=-t h(X, Y)
$$

which is also equivalent to

$$
A_{\omega X} Y=A_{\omega Y} X
$$

On the other hand, (3) and the symmetry of $F$ and $A$ lead to

$$
\begin{aligned}
g\left(A_{\omega X} Y, W\right) & =g(h(Y, W), \omega X)=g(h(Y, W), F X)=g\left(\bar{\nabla}_{W} Y, F X\right) \\
& =g\left(\bar{\nabla}_{W} F Y, X\right)=g\left(\bar{\nabla}_{W} \omega Y, X\right)=-g\left(A_{\omega Y} X, W\right),
\end{aligned}
$$

for any $X, Y \in \Gamma\left(T N_{\perp}\right)$ and $W \in \Gamma(T N)$, which implies that

$$
A_{\omega X} Y=-A_{\omega Y} X .
$$

From (23) and (24), we conclude that

$$
A_{\omega X} Y=0 \text { and } \operatorname{th}(X, Y)=0,
$$

for any $X, Y \in \Gamma(T N)$. Thus, from (22) and (25), we obtain

$$
g\left(h(U, X), \omega P_{2} U\right)=0 \quad \text { and } \quad g\left(h(X, Y), \omega P_{2} U\right)=0 .
$$


Furthermore, making use of (22), by direct calculations, we obtain

$$
A_{\omega P_{2} U} X=A_{\omega X} T P_{2} U=\operatorname{th}(X, U)=0 .
$$

From (20) and (26),

$$
\begin{aligned}
-X \ln (f) g\left(T P_{2} U, T P_{2} U\right) & =g\left(A_{\omega X} U, T P_{2} U\right)+g\left(\operatorname{th}(U, X), T P_{2} U\right) \\
& =g\left(h\left(U, T P_{2} U\right), \omega X\right)+g\left(\operatorname{th}(U, X), T P_{2} U\right) \\
& =g\left(\operatorname{th}(U, X), T P_{2} U\right)=0 .
\end{aligned}
$$

From (9) and (27) we conclude that

$$
X \ln (f) g\left(T P_{2} U, T P_{2} U\right)=X \ln (f) \cos ^{2} \theta g\left(P_{2} U, P_{2} U\right)=0 .
$$

Since $N_{\theta}$ is a proper slant submanifold, $g$ is a Riemannian metric and $P_{2} U$ is a nonzero vector, we can derive $X \ln (f)=0$, that is, the warping function $f$ is constant. Hence the proof is complete.

Conclusion 3.1. It is easy to see from Theorems 3.3 and 3.4 that there exist no warped product semi-slant submanifolds $N=N_{1} \times_{f} N_{\theta}$ in a locally Riemannian product manifold $M$ such that $N_{1}$ is invariant (anti-invariant) and $N_{\theta}$ is proper slant submanifold of $M$. But we can find the warped product semi-slant submanifolds $N=N_{\theta} \times{ }_{f} N_{T}$ (see Example 3.1) $\left(N=N_{\theta} \times{ }_{f} N_{\perp}\right.$ (see Example 3.2)) such that $N_{\theta}$ is proper slant and $N_{T}$ is invariant ( $N_{\perp}$ is anti-invariant) in a locally Riemannian product manifold $M$.

Next, to illustrate these cases, we shall give two examples.

EXAMPLE 3.1. Let $N$ be a submanifold of $\mathbb{R}^{8}=\mathbb{R}^{4} \times \mathbb{R}^{4}$ with coordinates $\left(x_{1}, x_{2}, x_{3}, x_{4}, x_{5}, x_{6}, x_{7}, x_{8}\right)$ given by

$$
\phi(\beta, \alpha, v, u)=(u+v, u-v, u \cos \alpha, u \sin \alpha, \sqrt{5} u, 2 v, u \cos \beta, u \sin \beta) .
$$

It is easy to see that the tangent bundle of $N$ is spanned by

$$
\begin{gathered}
Z_{1}=-u \sin \beta \frac{\partial}{\partial x_{7}}+u \cos \beta \frac{\partial}{\partial x_{8}}, \quad Z_{2}=-u \sin \alpha \frac{\partial}{\partial x_{3}}+u \cos \alpha \frac{\partial}{\partial x_{4}}, \\
Z_{3}=\frac{\partial}{\partial x_{1}}-\frac{\partial}{\partial x_{2}}+2 \frac{\partial}{\partial x_{6}}, \\
Z_{4}=\frac{\partial}{\partial x_{1}}+\frac{\partial}{\partial x_{2}}+\cos \alpha \frac{\partial}{\partial x_{3}}+\sin \alpha \frac{\partial}{\partial x_{4}}+\sqrt{5} \frac{\partial}{\partial x_{5}}+\cos \beta \frac{\partial}{\partial x_{7}}+\sin \beta \frac{\partial}{\partial x_{8}} .
\end{gathered}
$$

Then, with respect to the Riemannian product structure $F$ and usual metric tensor of $\mathbb{R}^{8}=\mathbb{R}^{4} \times \mathbb{R}^{4}, F(T N)$ becomes

$$
\begin{gathered}
F Z_{1}=-Z_{1}, \quad F Z_{2}=Z_{2}, \quad F Z_{3}=\frac{\partial}{\partial x_{1}}-\frac{\partial}{\partial x_{2}}-2 \frac{\partial}{\partial x_{6}} \\
Z_{4}=\frac{\partial}{\partial x_{1}}+\frac{\partial}{\partial x_{2}}+\cos \alpha \frac{\partial}{\partial x_{3}}+\sin \alpha \frac{\partial}{\partial x_{4}}-\sqrt{5} \frac{\partial}{\partial x_{6}}-\cos \beta \frac{\partial}{\partial x_{7}}-\sin \beta \frac{\partial}{\partial x_{8}} .
\end{gathered}
$$


It is easily to check that

$$
\cos ^{-1}\left(\frac{g\left(F Z_{3}, Z_{3}\right)}{\left\|F Z_{3}\right\| \cdot\left\|Z_{3}\right\|}\right)=\cos ^{-1}\left(\frac{g\left(F Z_{4}, Z_{4}\right)}{\left\|F Z_{4}\right\| \cdot\left\|Z_{4}\right\|}\right)=\cos ^{-1}\left(-\frac{1}{3}\right) .
$$

Then $N_{T}$ and $N_{\theta}$ can be taken as follows:

$$
T N_{T}=\operatorname{Span}\left\{Z_{1}, Z_{2}\right\} \quad \text { and } T N_{\theta}=\operatorname{Span}\left\{Z_{3}, Z_{4}\right\} .
$$

Thus $N_{\theta}$ is a slant submanifold with slant angle $\theta=\cos ^{-1}(-1 / 3)$. Furthermore, the metric tensor of $N=N_{T} \times{ }_{f} N_{\theta}$ is given by

$$
g_{N}=\left(6 d v^{2}+9 d u^{2}\right)+u^{2}\left(d \alpha^{2}+d \beta^{2}\right)=g_{N_{\theta}}+u^{2} g_{N_{T}} .
$$

Thus $N=N_{\theta} \times_{u^{2}} N_{T}$ is a warped product semi-slant submanifold of $\mathbb{R}^{8}$ with warping function $f=u$.

EXAMPLE 3.2. We consider the submanifold $N$ in $\mathbb{R}^{10}=\mathbb{R}^{4} \times \mathbb{R}^{6}$ given by

$$
\begin{aligned}
\varphi(u, v, \alpha)= & \left(\sqrt{3} u, \frac{2 k v}{\sqrt{k^{2}+1}}, u \cos \alpha,-u \sin \alpha,-u \cos \alpha,-u \sin \alpha,-k \sin u,\right. \\
& -k \sin v, k \cos u, k \cos v)
\end{aligned}
$$

where $k$ is a constant which is not zero. We can easily see that the tangent bundle of $N$ is spanned by vectors

$$
\begin{aligned}
Z_{1}= & \sqrt{3} \frac{\partial}{\partial x_{1}}+\cos \alpha \frac{\partial}{\partial x_{3}}-\sin \alpha \frac{\partial}{\partial x_{4}}-\cos \alpha \frac{\partial}{\partial x_{5}}-\sin \alpha \frac{\partial}{\partial x_{6}} \\
& -k \cos u \frac{\partial}{\partial x_{7}}-k \sin u \frac{\partial}{\partial x_{9}}, \\
Z_{2}= & \frac{2 k}{\sqrt{k^{2}+1}} \frac{\partial}{\partial x_{2}}-k \cos v \frac{\partial}{\partial x_{8}}-k \sin v \frac{\partial}{\partial x_{10}}, \\
Z_{3}= & -u \sin \alpha \frac{\partial}{\partial x_{3}}-u \cos \alpha \frac{\partial}{\partial x_{4}}+u \sin \alpha \frac{\partial}{\partial x_{5}}-u \cos \alpha \frac{\partial}{\partial x_{6}} .
\end{aligned}
$$

Since $F Z_{3}$ is orthogonal $T N$ and

$$
\theta=\cos ^{-1}\left(\frac{g\left(F Z_{1}, Z_{1}\right)}{\left\|Z_{1}\right\| \cdot\left\|F Z_{1}\right\|}\right)=\cos ^{-1}\left(\frac{g\left(F Z_{2}, Z_{2}\right)}{\left\|Z_{2}\right\| \cdot\left\|F Z_{2}\right\|}\right)=\cos ^{-1}\left(\frac{3-k^{2}}{5+k^{2}}\right),
$$

$N_{\perp}$ and $N_{\theta}$ can be taken as follows: $T N_{\perp}=\operatorname{Span}\left\{Z_{3}\right\}$ is an anti-invariant distribution and $T N_{\theta}=\operatorname{Span}\left\{Z_{1}, Z_{2}\right\}$ is a slant distribution. Here $F$ and $g$ denote the Riemannian product structure and usual metric tensor of $\mathbb{R}^{10}=\mathbb{R}^{4} \times \mathbb{R}^{6}$, respectively. Furthermore, the metric tensor of $N=N_{\theta} \times{ }_{f} N_{\perp}$ is given by

$$
g_{N}=\left(5+k^{2}\right) d u^{2}+\left(\frac{k^{4}+5 k^{2}}{k^{2}+1}\right) d v^{2}+2 u^{2} d \alpha^{2}=g_{N_{\theta}}+2 u^{2} g_{N_{\perp}} .
$$


Thus $N=N_{\theta} \times{ }_{\sqrt{2} u} N_{\perp}$ is a warped product semi-slant submanifold with slant angle $\theta=\cos ^{-1}\left(\left(3-k^{2}\right) /\left(5+k^{2}\right)\right)$ and warping function $f=\sqrt{2} u$.

\section{References}

[1] A. Bejancu, 'Semi-invariant submanifolds of locally product Riemannian manifold', Ann. Univ. Timisoara S. Math. XXII (1984), 3-11.

[2] J. L. Cabrerizo, A. Carriazo, L. M. Fernandez and M. Fernandez, 'Semi-slant submanifolds of a Sasakian manifold', Geom. Dedicata 78 (1999), 183-199.

[3] B.-Y. Chen, 'Slant submanifolds of complex projective and complex hyperbolic spaces', Glasg. Math. J. 42 (2000), 439-454.

[4] B.-Y. Chen, 'Geometry of warped product CR-submanifolds in Kaehler manifolds', Monatsh. Math. 133 (2001), 177-195.

[5] B.-Y. Chen, 'CR-warped products in complex projective spaces with compact holomorphic factor', Monatsh. Math. 141 (2004), 177-186.

[6] B.-Y. Chen, Geometry of slant submanifolds (Katholieke Universiteit Leuven, Leuven, 1990).

[7] A. Lotto, 'Slant submanifolds in contact geometry', Bull. Math. Soc. Roumanie 39 (1996), 183198.

[8] K. Matsumoto and I. Mihai, 'Warped product submanifolds in Sasakian space forms', SUT J. Math. 38(2) (2002), 135-144.

[9] K. Matsumoto, 'On submanifolds of locally product Riemannian manifolds', TRU Math. 18(2) (1982), 145-157.

[10] B. O'Neill, Semi-Riemannian geometry with applications to relativity (Academic Press, New York, 1983).

[11] S. Tachibana, 'Some theorems on a locally product Riemannian manifold', Tohoku Math. J. 12 (1960), 281-292.

[12] H. Li and X. Li, 'Semi-slant submanifolds of locally product manifold', Georgian Math. J. 12(2) (2005), 273-282.

\footnotetext{
MEHMET ATÇEKEN, GOP University, Faculty of Arts and Sciences, Department of Mathematics, 60200 Tokat, Turkey

e-mail: matceken@gop.edu.tr
} 